\title{
Real-World Experience of Safety of Mycophenolate Mofetil in 119 Japanese Patients with Systemic Lupus Erythematosus: A Retrospective Single-Center Study
}

\author{
Yoshiyuki Abe $(D$, Kurisu Tada, Ken Yamaji, and Naoto Tamura \\ Department of Internal Medicine and Rheumatology, Juntendo University School of Medicine, Tokyo, Japan \\ Correspondence should be addressed to Yoshiyuki Abe; yo-abe@juntendo.ac.jp
}

Received 2 August 2020; Revised 11 January 2021; Accepted 15 January 2021; Published 25 January 2021

Academic Editor: Vinod Chandran

Copyright (C) 2021 Yoshiyuki Abe et al. This is an open access article distributed under the Creative Commons Attribution License, which permits unrestricted use, distribution, and reproduction in any medium, provided the original work is properly cited.

\begin{abstract}
Objectives. Mycophenolate mofetil (MMF) is the standard treatment for lupus nephritis. In Japan, it was approved for lupus nephritis in 2015. We investigated its real-world safety and effectiveness in Japanese patients with systemic lupus erythematosus (SLE). Methods. We analyzed the continuation rate, adverse events, and reasons for discontinuation of MMF in Japanese patients with SLE in a retrospective single-center study. We included 119 patients who received MMF from 31 July 2015 to 31 May 2019. To compare demographic and clinical characteristics between groups, the Mann-Whitney $U$-test was used for nonnormally distributed variables. Categorical variables were compared using Fisher's exact test. Kaplan-Meier curves were plotted for the discontinuation rate of MMF. Results. Patients consisted of 18 males and 101 females. Thirty-five patients discontinued MMF. The cumulative discontinuation rate was $42.4 \%$. Twenty-nine patients discontinued MMF due to adverse events, and six patients discontinued MMF due to remission of SLE or desire for childbearing. At the time of the last observation, the lupus low disease activity state achievement rate was significantly lower in patients who experienced adverse events than those who did not $(64 \%$ vs. $35 \%, P=0.009)$. We examined the concentration of mycophenolate acid (trough level) in stored frozen serum in 11 patients. Two patients had irreversible complications due to viral meningitis; their trough mycophenolate acid concentrations were 8.3 and $6.3 \mu \mathrm{g} / \mathrm{mL}$, respectively. Conclusions. Although MMF may be effective in Japanese patients with SLE, physicians should pay attention to infections in patients with high mycophenolate acid concentrations.
\end{abstract}

\section{Introduction}

Mycophenolate mofetil (MMF) is an immunosuppressant that was approved for the prevention of transplant rejection in 1992 [1]. MMF is an ester of mycophenolate acid (MPA), the active metabolite. Since MPA inhibits de novo purine synthesis, on which only lymphocytes depend, MPA has specific antiproliferative effects [2]. The MMF is used as an immunosuppressant to treat rheumatic diseases, especially lupus nephritis [3, 4]. The American College of Rheumatology (ACR) and European League Against Rheumatism (EULAR) published guidelines for lupus nephritis in 2012 in which they recommended that MMF and cyclophosphamide should be considered equivalent for patients with International Society of Nephrology class III/IV lupus nephritis [5-7]. MMF is currently an important treatment for systemic lupus erythematosus (SLE).
MMF often causes adverse events. Typical adverse events include infections, upper gastrointestinal symptoms, and diarrhea [5]. ACR guidelines recommend that "Asians compared to non-Asians might require lower doses of MMF for similar efficacy" [7-9]. Previous Japanese studies have reported MMF doses of almost 1,000-2,000 mg/day/person for lupus nephritis [10-14]. In this single-center retrospective study, we evaluated medication continuation, adverse events, and reasons for discontinuation of MMF in 119 patients with SLE.

\section{Patients and Methods}

2.1. Patients. This retrospective single-center observational study included 173 patients who received MMF treatment from 31 July 2015 to 31 May 2019 at Juntendo University Hospital. MMF was approved for SLE on 31 July 2015 in 
Japan. We ultimately analyzed 119 of the initial 173 patients. We excluded 54 patients because 8 patients started MMF before 31 July 2015, 20 patients had been treated with MMF at another hospital, and 26 patients were treated with MMF for other rheumatic diseases. All 119 patients were diagnosed with SLE according to the 1997 ACR SLE classification criteria [15]. There was no standardized treatment regimen in this retrospective observational study; MMF treatment was determined by each patient's physician. The ethics committee of Juntendo University Hospital approved this study (approval number 19-054). Patients could opt out of the study through the hospital's website.

2.2. Clinical Evaluations and Outcomes. Clinical data, including patient demographics, clinical manifestations, laboratory data, outcomes, adverse events, and reasons for MMF discontinuation, were obtained from medical records. We analyzed clinical manifestations from any point in time. Laboratory data were from the time of MMF initiation. Treatments included all therapies received by patients at the time of MMF initiation and history of treatments before starting MMF. The primary outcome was the rate of MMF continuation. Secondary outcomes were adverse events associated with MMF, reasons for MMF discontinuation, MMF dose during the last observation period, and prevalence of the lupus low disease activity state (LLDAS) during the last observation period [16]. In this study, a major SLE flare was defined as British Isles Lupus Assessment Group Index category A disease [17].

2.3. Measurement of Serum MPA Levels. Serum MPA levels were measured retrospectively with frozen serum. We stored surplus serum after clinical laboratory testing for retrospective testing (Juntendo University Hospital ethics committee approval number 334). All measurements were performed using the enzyme multiplied immunoassay technique on serum samples collected when the MMF dose had been stable for more than a week. In order to collect serum at precisely 12 hours after administration to assess trough levels, only serum samples from hospitalized patients with confirmed blood collection times were selected.

2.4. Statistical Analysis. To compare demographic and clinical characteristics between groups, the Mann-Whitney $U$-test was used for nonnormally distributed variables. Categorical variables were compared using Fisher's exact test. Spearman's correlation coefficients were calculated. Kaplan-Meier curves were plotted for the discontinuation rate of MMF. Data are presented as medians (interquartile range (IQR)). Analyses were performed using SPSS version 23.0 software (SPSS, Armonk, $\mathrm{NY}$ ) with $P<0.05$ considered to be statistically significant.

\section{Results}

The median observation period was 16 (5-33) months. Patients consisted of 18 males and 101 females. The median age was 38 (31-46) years, and the median duration of SLE was 138 (39-242) months. Ninety-six patients received MMF as induction therapy and 23 patients received MMF as maintenance therapy. MMF was used for the following reasons: lupus nephritis (70 patients), serological abnormality
(24 patients), change from another immunosuppressant (10 patients), rash (8 patients), neuropsychiatric manifestation (3 patients), cytopenia ( 2 patients), and arthritis (2 patients). Thirty-five patients discontinued MMF. The cumulative discontinuation rate was $42.4 \%$. Twenty-nine of 35 patients discontinued MMF because of adverse events and six patients discontinued due to SLE remission or desire for childbearing. The reasons for discontinuation were as follows: infection (11 patients), nausea or diarrhea (9 patients), SLE exacerbation (3 patients), SLE remission (3 patients), desire for childbearing (3 patients), cytopenia (2 patients), and renal dysfunction, liver dysfunction, alopecia, and rash (1 patient each) (Figure 1(a)). For all adverse events, infection and SLE exacerbation were differentiated from general adverse events. Nausea or diarrhea, cytopenia, renal dysfunction, liver dysfunction, alopecia, and rash were distinguished from adverse effects of MMF. Table 1 shows the background characteristics, laboratory test findings, treatments, and outcomes in all patients and by adverse event status. The group with adverse events had lower hemoglobin, as well as higher alanine aminotransferase (ALT) and blood urea nitrogen (BUN) levels. At the last observation, the LLDAS achievement rate was significantly lower in the adverse event group than in the no-adverse event group (64\% vs. 35\%; $P=0.009)$. Supplemental Table 1 shows the comparison between patients who received MMF as induction therapy versus maintenance therapy. The induction therapy group had lower concentrations of complement and higher antideoxyribonucleic acid (DNA) antibody titers and glucocorticoid (GC) dose at the start of MMF therapy. Supplemental Tables 2 and 3 show the comparison between patients with general adverse events versus adverse effects associated with MMF. Among patients with general adverse events, serum aspartate aminotransferase levels were higher. There were no significant differences in other variables.

At the time of LLDAS achievement, patients in the no-AE and AE groups had a mean daily GC dose of $7.0(6.0-7.0) \mathrm{mg}$ and $7.0(7.0-7.13) \mathrm{mg}(P=0.27)$, respectively. The proportion of patients using MMF in the two groups was $93 \%$ and $0 \%$ (not applicable), respectively. The proportion of patients using tacrolimus in the two groups was $46 \%$ and $20 \%$ $(P=0.17)$, respectively. There was one patient treated with azathioprine in the no-AE group and one patient treated with belimumab in the AE group.

Figure 1(b) shows the Kaplan-Meier curve for the overall MMF discontinuation rate. Figure 1(c) shows the KaplanMeier curve for the MMF discontinuation rate due to adverse events. Fifty-five percent of adverse events occurred in the first 2 months after the start of MMF therapy, and $79 \%$ occurred in the first 6 months. Figure 1(d) shows the MMF dose at the last observation. The median MMF dose at the last observation was $1,000(1,000-1,500) \mathrm{mg}$.

Seven severe infections occurred, consisting of two cases of varicella-zoster virus (VZV) meningitis, and one case each of disseminated VZV, urosepsis, osteomyelitis of the mandible, necrotizing fasciitis, and multiple subcutaneous abscesses. The GC dose in each patient at the time of infection was $60 \mathrm{mg}, 55 \mathrm{mg}, 55 \mathrm{mg}, 35 \mathrm{mg}, 30 \mathrm{mg}, 28 \mathrm{mg}$, and $14 \mathrm{mg}$ daily, respectively. One patient with disseminated VZV died during the observation period. There were four mild infections 


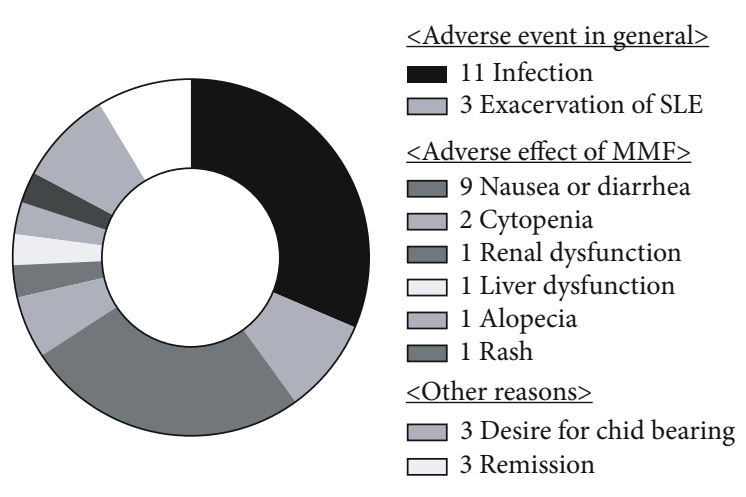

(a)

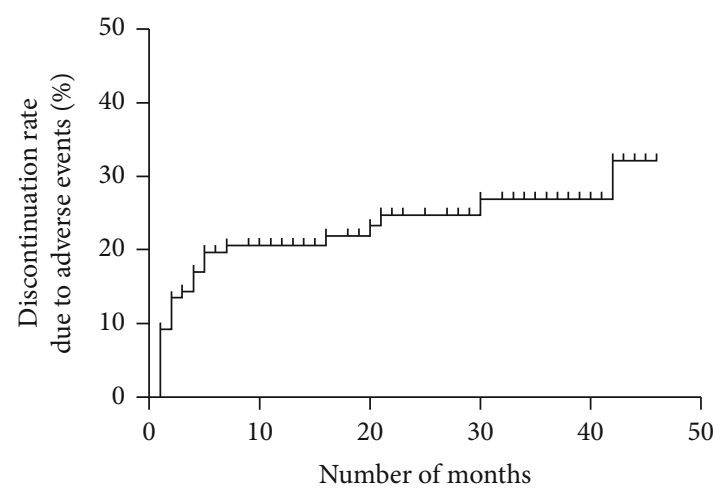

(c)

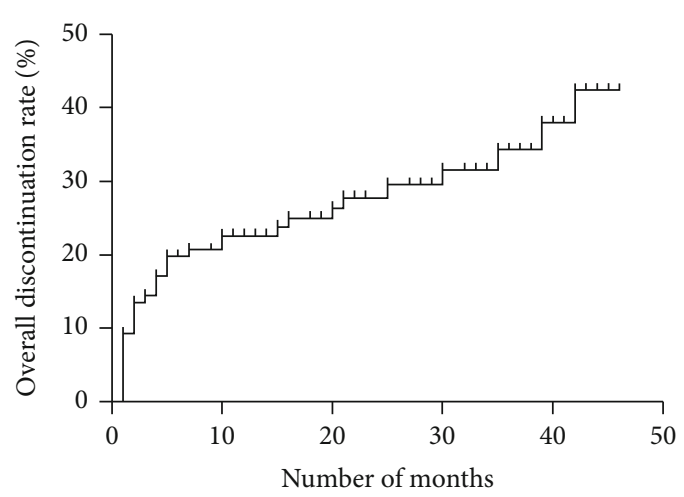

(b)

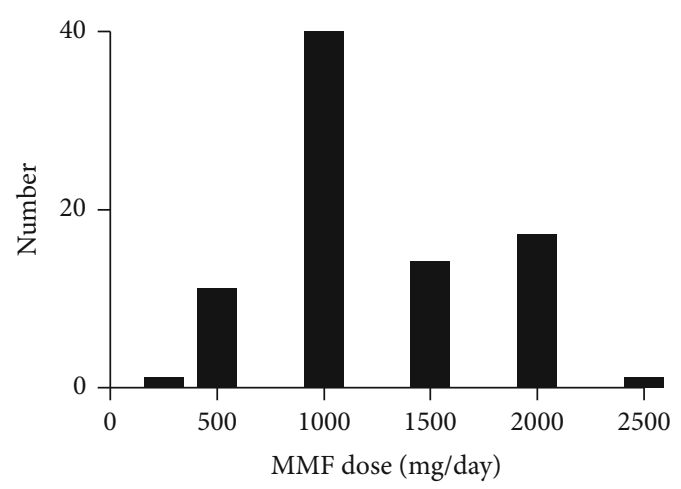

(d)

Figure 1: Details about MMF use. (a) Distribution of reasons for MMF discontinuation. (b) The Kaplan-Meier curve for MMF discontinuation. (c) The Kaplan-Meier curve for MMF discontinuation due to adverse events. (d) MMF dose at last observation. MMF: mycophenolate mofetil.

consisting of upper respiratory infections and mycobacterial dermatitis. Supplemental Table 4 shows the details of immunosuppressive therapies and outcomes in each patient.

We examined serum trough MPA concentrations (Table 2) in our patients' surplus frozen serum samples, which were stored as a general practice. All 11 patients received MMF as induction therapy. Reliable trough levels could only be measured in 11 samples. It was not possible to measure trough levels in the other samples because the timing of blood collection and oral MMF administration was uncertain. Two patients who had irreversible brain damage due to viral meningitis had MPA concentrations of 8.3 and $6.3 \mu \mathrm{g} / \mathrm{mL}$, respectively.

\section{Discussion}

We analyzed 119 patients with SLE treated with MMF. The overall discontinuation rate was $42.4 \%$, the adverse eventrelated discontinuation rate was $34.0 \%$, and there were 29 adverse events. Although the most reason for discontinuation was 11 infection, we considered that it was also influenced by the high median GC of $20(10-40) \mathrm{mg} / \mathrm{dL}$. These findings correspond to real-world safety data for MMF in Japanese patients with SLE.

The effectiveness of MMF for patients with SLE was also revealed in our study. The LLDAS achievement rate was $57 \%$. Several patients were still tapering from GCs at the last obser- vation, so the prevalence of LLDAS might have been higher if the observation period was extended. However, the interpretation of these results was limited due to the lack of standard therapeutic regimens and uniform follow-up duration. The no-AE group had a significantly higher LLDAS achievement rate, which might indicate the effectiveness of $M M F$ in Japanese patients with SLE.

We analyzed the safety of MMF in Japanese patients with SLE. Life-threatening adverse events included seven severe infections. In order to use MMF safely, we consider it necessary to analyze factors that might predict adverse events. The analysis showed that lower hemoglobin, higher BUN, and use of other immunosuppressants are associated with adverse events. However, anemia was not associated with serum MPA levels in a previous report [18]. These results may not reflect renal dysfunction, because only BUN was associated with adverse events, not creatinine, and eGFR. Unfortunately, these results may include confounding factors because hemoglobin, BUN, and use of other immunosuppressants are correlated with each other and other factors. Focusing on the trends of higher anti-DNA antibodies, proteinuria, SLE disease activity index, and lower complement concentrations instead of anemia and BUN suggests that the AE group may have more active SLE. However, no statistical differences were observed; these findings should be verified in a larger study. We found MPA levels of 8.3 and $6.3 \mu \mathrm{g} / \mathrm{mL}$, respectively, in two patients with VZV meningitis. MMF was associated with increased 
TABLE 1: Characteristics of patients in the AE and no-AE groups.

\begin{tabular}{|c|c|c|c|c|}
\hline & $\begin{array}{l}\text { Overall } \\
N=119\end{array}$ & $\begin{array}{c}\text { No-AE group } \\
\quad n=90\end{array}$ & $\begin{array}{c}\text { AE group } \\
n=29\end{array}$ & $P$ value \\
\hline Age at start of MMF therapy, median (IQR) & $38(31-46)$ & $39(31-46)$ & $33(29-45)$ & 0.20 \\
\hline Female sex, $n(\%)$ & $101(85)$ & $74(82)$ & $27(93)$ & 0.23 \\
\hline Body weight (kg), median (IQR) & $55(48-63)$ & $57(48-64)$ & $54(46-62)$ & 0.23 \\
\hline Duration of SLE, months, median (IQR) & $138(39-242)$ & $133(40-227)$ & $160(23-262)$ & 0.68 \\
\hline MMF started as induction therapy, $n(\%)$ & $96(81)$ & $72(81)$ & $24(83)$ & 1.00 \\
\hline Malar rash, $n(\%)$ & $62(52)$ & $51(57)$ & $11(38)$ & 0.09 \\
\hline Discoid rash, $n(\%)$ & $23(20)$ & $16(18)$ & $7(24)$ & 0.59 \\
\hline Photosensitivity, $n$ (\%) & $31(26)$ & $21(24)$ & $10(35)$ & 0.15 \\
\hline Oral ulcers, $n(\%)$ & $31(27)$ & $20(23)$ & $11(38)$ & 0.33 \\
\hline Arthritis, $n(\%)$ & $75(64)$ & $54(61)$ & $21(72)$ & 0.28 \\
\hline Serositis, $n(\%)$ & $28(24)$ & $23(26)$ & $5(17)$ & 0.45 \\
\hline Renal disorder, $n(\%)$ & $81(68)$ & $62(69)$ & $19(66)$ & 0.82 \\
\hline Neurologic disorder, $n(\%)$ & $9(8)$ & $6(7)$ & $3(10)$ & 0.69 \\
\hline Hematologic disorder, $n(\%)$ & $89(75)$ & $68(76)$ & $21(72)$ & 0.8 \\
\hline Immunologic disorder, $n(\%)$ & $118(99)$ & $89(99)$ & $29(100)$ & 1.00 \\
\hline Antinuclear antibody, $n(\%)$ & $119(100)$ & $90(100)$ & $29(100)$ & N/A \\
\hline SLEDAI at start of MMF therapy, median (IQR) & $6(4-10)$ & $6(4-10)$ & $8(4-13)$ & 0.14 \\
\hline Number of SLE flare-ups, median (IQR) & $1(1-3)$ & $2(1-2)$ & $1(0-3)$ & 0.30 \\
\hline WBC count $(/ \mu \mathrm{L})$, median $(\mathrm{IQR})$ & $6,600(4,500-7,900)$ & $6,100(4,400-7,600)$ & $7,000(5,800-9,500)$ & 0.07 \\
\hline Lymphocytes $(/ \mu \mathrm{L})$, median (IQR) & $1,001(598-1,335)$ & $925(544-1,326)$ & $1,039(619-1,404)$ & 0.41 \\
\hline Hemoglobin (g/dL), median (IQR) & $11.8(10.6-13.1)$ & $12.1(11.1-13.2)$ & $11.2(10-12.3)$ & $0.014^{*}$ \\
\hline Platelets $\left(10^{4} / \mu \mathrm{L}\right)$, median (IQR) & $23.3(18.6-28.8)$ & $23.6(19.3-28)$ & $22.4(17.8-30.7)$ & 0.91 \\
\hline AST (U/L), median (IQR) & $19(15-24)$ & $18(15-24)$ & $19(15-25)$ & 0.67 \\
\hline ALT (U/L), median (IQR) & $18(12-28)$ & $16(11-25)$ & $25(15-34)$ & 0.011 \\
\hline Albumin (g/dL), median (IQR) & $3.5(3-3.9)$ & $3.6(3.1-3.9)$ & $3.2(2.6-3.8)$ & 0.06 \\
\hline BUN (mg/dL), median (IQR) & $15(11-20)$ & $14(10-19)$ & $18(14-27)$ & $0.006^{* *}$ \\
\hline Creatinine (mg/dL), median (IQR) & $0.62(0.49-0.83)$ & $0.62(0.49-0.78)$ & $0.57(0.48-0.99)$ & 0.48 \\
\hline eGFR $\left(\mathrm{mL} / \mathrm{min} / 1.73 \mathrm{~m}^{2}\right)$, median $(\mathrm{IQR})$ & $91(67-115)$ & $92(70-115)$ & $89(46-124)$ & 0.63 \\
\hline CH50 (U/mL), median (IQR) & $28(20-38)$ & $29(21-39)$ & $25(16-37)$ & 0.33 \\
\hline C3 (mg/dL), median (IQR) & $66(48-84)$ & $69(52-85)$ & $61(44-83)$ & 0.38 \\
\hline C4 (mg/dL), median (IQR) & $11(7-18)$ & $12(7-19)$ & $10(5-17)$ & 0.31 \\
\hline IgG (mg/dL), median (IQR) & $1,220(914-1,455)$ & $1,243(943-1,431)$ & $1,151(597-1,718)$ & 0.59 \\
\hline Anti-DNA antibody (RIA) (IU/mL), median (IQR) & $11(0-62)$ & $7(0-50)$ & $30(5-188)$ & 0.053 \\
\hline Anti-U1-RNP antibody, positivity, $n(\%)$ & $43(36)$ & $34(38)$ & $9(31)$ & 0.52 \\
\hline Anti-Sm antibody, positivity, $n(\%)$ & $16(14)$ & $14(16)$ & $2(7)$ & 0.35 \\
\hline Anti-CL antibody, positivity, $n(\%)$ & $26(22)$ & $22(25)$ & $4(14)$ & 0.30 \\
\hline Anti-CL $\beta 2$ GP1 antibody, positivity, $n(\%)$ & $13(11)$ & $9(10)$ & $4(14)$ & 1.00 \\
\hline Lupus anticoagulant, median (IQR) & $0.9(0.9-1)$ & $1(0.9-1)$ & $0.9(0.8-1)$ & 0.51 \\
\hline Proteinuria (g/day), median (IQR) & $0.6(0-2.3)$ & $0.5(0-2.1)$ & $0.7(0-2.5)$ & 0.46 \\
\hline Hematuria, positivity, $n(\%)$ & $38(33)$ & $28(33)$ & $10(35)$ & 1.00 \\
\hline GC dose at start of MMF therapy (mg/day), median (IQR) & $20(10-40)$ & $19(10-36)$ & $28(12-55)$ & 0.07 \\
\hline Overall maximum GC dose (mg/day), median (IQR) & $50(40-60)$ & $50(40-60)$ & $50(40-60)$ & 0.93 \\
\hline LLDAS at last observation, $n(\%)$ & $67(57)$ & $57(64)$ & $10(35)$ & $0.009^{* *}$ \\
\hline
\end{tabular}

AE: adverse event; MMF: mycophenolate mofetil; IQR: interquartile range; SLE: systemic lupus erythematosus; SLEDAI: SLE disease activity index; WBC: white blood cell; AST: aspartate aminotransferase; ALT: alanine aminotransferase; BUN: blood urea nitrogen; eGFR: estimated glomerular filtration rate; IgG: immunoglobulin G; DNA: deoxyribonucleic acid; RIA: radioimmunoassay; U1RNP: U1-ribonucleoprotein; Sm: Smith; CL: cardiolipin; CL $\beta$ 2GP1: cardiolipin $\beta 2$-glycoprotein I; GC: glucocorticoid; LLDAS: lupus low disease activity state. ${ }^{*} P<0.05 .{ }^{* *} P<0.01$. 
TABLE 2: Characteristics of patients with measured MPA trough levels.

\begin{tabular}{|c|c|c|c|c|c|c|c|c|c|}
\hline Patient & $\begin{array}{l}\text { Age at start of } \\
\text { MMF therapy } \\
\quad \text { (years) }\end{array}$ & Sex & $\begin{array}{l}\text { Duration of } \\
\text { MMF therapy } \\
\text { (month) }\end{array}$ & $\begin{array}{c}\text { MMF } \\
\text { trough level } \\
(\mu \mathrm{g} / \mathrm{mL})\end{array}$ & $\begin{array}{c}\text { MMF } \\
\text { dose } \\
\text { (mg/day) }\end{array}$ & $\begin{array}{c}\text { SLEDAI at } \\
\text { start of MMF } \\
\text { therapy }\end{array}$ & $\begin{array}{c}\text { Outcome of } \\
\text { MMF }\end{array}$ & $\begin{array}{c}\text { Adverse } \\
\text { event }\end{array}$ & $\begin{array}{c}\text { Achievement } \\
\text { of LLDAS }\end{array}$ \\
\hline 1 & 32 & Male & 5 & 8.5 & 2,000 & 14 & Withdrawal & $\begin{array}{c}\text { Renal } \\
\text { dysfunction }\end{array}$ & No \\
\hline 2 & 43 & Female & 16 & 8.3 & 2,000 & 12 & Withdrawal & $\begin{array}{c}\text { Infection } \\
\text { (VZV } \\
\text { meningitis) }\end{array}$ & Yes \\
\hline 3 & 38 & Female & 1 & 7.3 & 1,500 & 18 & $\begin{array}{c}\text { Dose } \\
\text { decrease }\end{array}$ & & No \\
\hline 4 & 43 & Female & 2 & 6.7 & 2,000 & 14 & Withdrawal & Cytopenia & No \\
\hline 5 & 46 & Male & 20 & 6.6 & 2,000 & 6 & $\begin{array}{c}\text { Dose } \\
\text { decrease }\end{array}$ & & Yes \\
\hline 6 & 32 & Female & 4 & 6.3 & 2,000 & 14 & Withdrawal & $\begin{array}{c}\text { Infection } \\
\text { (VZV } \\
\text { meningitis) }\end{array}$ & No \\
\hline 7 & 32 & Female & 2 & 5.3 & 1,500 & 16 & $\begin{array}{c}\text { Dose } \\
\text { decrease }\end{array}$ & & No \\
\hline 8 & 18 & Female & 12 & 4.7 & 2,000 & 12 & $\begin{array}{c}\text { Dose } \\
\text { decrease }\end{array}$ & & No \\
\hline 9 & 38 & Female & 11 & 4 & 1,000 & 9 & Continue & & No \\
\hline 10 & 23 & Female & 3 & 3.5 & 2,000 & 8 & Withdrawal & Alopecia & Yes \\
\hline 11 & 23 & Female & 1 & 3.1 & 2,000 & 20 & Withdrawal & Cytopenia & No \\
\hline
\end{tabular}

MPA: mycophenolate acid; MMF: mycophenolate mofetil; SLEDAI: systemic lupus erythematosus disease activity index; LLDAS: lupus low disease activity state.

susceptibility to VZV infection in previous studies on kidney transplantation $[19,20]$ and SLE $[21,22]$. There is a Japanese case report of SLE and fatal VZV infection [23]. Physicians should be aware of the risk of viral infections such as VZV in patients with SLE taking MMF.

In this study, the median dose of MMF at the last observation was $1,000 \mathrm{mg}$, reflecting physicians' real-world choices for SLE maintenance therapy in Japan. EULAR recommends $3,000 \mathrm{mg}$ of $\mathrm{MMF}$ for induction therapy and 2,000 $\mathrm{mg}$ of MMF for maintenance remission therapy in non-Asian patients with SLE and 2,000 mg of MMF for induction therapy in Asian patients with SLE. Because GCs interfere with MPA bioavailability, patients had higher MPA concentrations while being tapered off GCs after induction therapy than during induction therapy [24].

The usefulness of therapeutic drug monitoring for MPA in patients with SLE is controversial. Several studies on SLE reported that the concentration of MPA is associated with therapeutic effect and adverse events [25-27], only therapeutic effects [28-36], or neither [13]. Higher MPA concentration is associated with effectiveness in lupus nephritis during therapy to induce remission. Actual mean daily MMF doses were appropriate 1,500-2,000 mg [13, 27-29, $31,32]$. In these studies, the mean predose MPA concentration was between 1.7 and $2.5 \mu \mathrm{g} / \mathrm{mL}$. Higher MPA concentration during maintenance therapy is associated with favorable outcomes [13, 25-27, 31, 33-36]. The mean daily MMF dose was $1,900-2,000 \mathrm{mg}$, and the mean predose MPA concentration was between 1.7 and $4.2 \mu \mathrm{g} / \mathrm{mL}$. Trough levels did not correspond to the area under the blood concentration-time curve in patients with SLE $[13,25]$. We could not conclude that therapeutic drug monitoring was useful. Further evaluation is needed.

A noteworthy point of our study was that MPA levels were measured retrospectively. If we had confirmed high serum MPA levels during treatment, we may have reduced the dose of MMF. Due to the difficulties in reliably measuring trough levels in a retrospective study, serum trough MPA levels could only be examined in 11 patients. This was the major limitation of our study.

\section{Conclusion}

We evaluated the rate of MMF continuation and reasons for MMF discontinuation in Japanese patients with SLE. High serum MPA levels may be associated with severe infections in Japanese patients with SLE. Although MMF may be effective in Japanese patients with SLE, physicians should pay attention to infections in patients with high MPA concentrations.

\section{Data Availability}

The data used to support the findings of this study are available from the corresponding author upon request.

\section{Conflicts of Interest}

YA, KT, and KY have no conflicts of interest to declare. 


\section{Acknowledgments}

The authors would like to thank Dr. Yuki Asai for assistance with data collection. NT has received research grants from Chugai Pharmaceutical Co., Ltd.

\section{Supplementary Materials}

Supplementary Table 1: characteristics of patients taking MMF as induction versus maintenance therapy. Supplementary Table 2: characteristics of patients with had general AEs versus adverse effects associated with MMF. Supplementary Table 3: patient characteristics by reason for MMF discontinuation. Supplementary Table 4: characteristics of patients who discontinued MMF due to severe infection. (Supplementary Materials)

\section{References}

[1] H. W. Sollinger, F. O. Belzer, M. H. Deierhoi et al., "RS-61443 (mycophenolate mofetil). A multicenter study for refractory kidney transplant rejection," Annals of Surgery, vol. 216, no. 4, pp. 513-518, 1992.

[2] J. J. Lipsky, "Mycophenolate mofetil," The Lancet, vol. 348, no. 9038, pp. 1357-1359, 1996.

[3] M. A. Dooley, F. G. Cosio, P. H. Nachman et al., "Mycophenolate mofetil therapy in lupus nephritis: clinical observations," Journal of the American Society of Nephrology, vol. 10, no. 4, pp. 833-839, 1999.

[4] M. Gaubitz, A. Schorat, H. Schottel, P. Kern, and W. Domschke, "Mycophenolate mofetil for the treatment of systemic lupus erythematosus: an open pilot trial," Lupus, vol. 8, no. 9, pp. 731-736, 1999.

[5] E. M. Ginzler, M. A. Dooley, C. Aranow et al., "Mycophenolate mofetil or intravenous cyclophosphamide for lupus nephritis," The New England Journal of Medicine, vol. 353, no. 21, pp. 2219-2228, 2005.

[6] G. K. Bertsias, M. Tektonidou, Z. Amoura et al., "Joint European League Against Rheumatism and European Renal Association-European Dialysis and Transplant Association (EULAR/ERA-EDTA) recommendations for the management of adult and paediatric lupus nephritis," Annals of the Rheumatic Diseases, vol. 71, no. 11, pp. 1771-1782, 2012.

[7] B. H. Hahn, M. McMahon, A. Wilkinson et al., "American College of Rheumatology guidelines for screening, treatment, and management of lupus nephritis," Arthritis Care \& Research, vol. 64, no. 6, pp. 797-808, 2012.

[8] Z. Touma, D. D. Gladman, M. B. Urowitz, J. Beyene, E. M. Uleryk, and P. S. Shah, "Mycophenolate mofetil for induction treatment of lupus nephritis: a systematic review and metaanalysis," The Journal of Rheumatology, vol. 38, no. 1, pp. 69-78, 2011.

[9] M. Y. Weng, C. T. Weng, and M. F. Liu, "The efficacy of lowdose mycophenolate mofetil for treatment of lupus nephritis in Taiwanese patients with systemic lupus erythematosus," Clinical Rheumatology, vol. 29, no. 7, pp. 771-775, 2010.

[10] R. Hara, H. Miyazawa, K. Nishimura et al., "A national survey on current use of mycophenolate mofetil for childhood-onset systemic lupus erythematosus in Japan," Modern Rheumatology, vol. 25, no. 6, pp. 858-864, 2015.
[11] H. Ikeuchi, K. Hiromura, S. Takahashi et al., "Efficacy and safety of multi-target therapy using a combination of tacrolimus, mycophenolate mofetil and a steroid in patients with active lupus nephritis," Modern Rheumatology, vol. 24, no. 4, pp. 618-625, 2014.

[12] A. Onishi, D. Sugiyama, G. Tsuji et al., "Mycophenolate mofetil versus intravenous cyclophosphamide for induction treatment of proliferative lupus nephritis in a Japanese population: a retrospective study," Modern Rheumatology, vol. 23, no. 1, pp. 89-96, 2014.

[13] T. Katsuno, T. Ozaki, T. Ozeki et al., "Investigation on the benefits of mycophenolate mofetil and therapeutic drug monitoring in the treatment of Japanese patients with lupus nephritis," Clinical and Experimental Nephrology, vol. 22, no. 6, pp. 13411350, 2018.

[14] M. Kawazoe, K. Kaneko, Z. Yamada et al., "Efficacy of mycophenolate mofetil in Japanese patients with systemic lupus erythematosus," Clinical Rheumatology, vol. 38, no. 6, pp. 15711578, 2019.

[15] M. C. Hochberg, "Updating the American College of Rheumatology revised criteria for the classification of systemic lupus erythematosus," Arthritis and Rheumatism, vol. 40, no. 9, p. $1725,1997$.

[16] K. Franklyn, C. S. Lau, S. V. Navarra et al., "Definition and initial validation of a Lupus Low Disease Activity State (LLDAS)," Annals of the Rheumatic Diseases, vol. 75, no. 9, pp. 16151621, 2016.

[17] D. A. Isenberg and C. Gordon, "From BILAG to BLIPS-disease activity assessment in lupus past, present and future," Lupus, vol. 9, no. 9, pp. 651-654, 2000.

[18] L. J. Langman, D. F. LeGatt, and R. W. Yatscoff, "Blood distribution of mycophenolic acid," Therapeutic Drug Monitoring, vol. 16, no. 6, pp. 602-607, 1994.

[19] R. Lauzurica, B. Bayés, C. Frías et al., "Disseminated varicella infection in adult renal allograft recipients: role of mycophenolate mofetil," Transplantation Proceedings, vol. 35, no. 5, pp. 1758-1759, 2003.

[20] W. S. Rothwell, J. M. Gloor, B. Z. Morgenstern, and D. S. Milliner, "Disseminated varicella infection in pediatric renal transplant recipients treated with mycophenolate mofetil," Transplantation, vol. 68, no. 1, pp. 158-161, 1999.

[21] E. F. Chakravarty, K. Michaud, R. Katz, and F. Wolfe, "Increased incidence of herpes zoster among patients with systemic lupus erythematosus," Lupus, vol. 22, no. 3, pp. 238-244, 2013.

[22] C. Rondaan, A. de Haan, G. Horst et al., "Altered cellular and humoral immunity to varicella-zoster virus in patients with autoimmune diseases," Arthritis \& Rhematology, vol. 66, no. 11, pp. 3122-3128, 2014.

[23] M. Habuka, Y. Wada, Y. Kurosawa et al., "Fatal visceral disseminated varicella zoster infection during initial remission induction therapy in a patient with lupus nephritis and rheumatoid arthritis-possible association with mycophenolate mofetil and high-dose glucocorticoid therapy: a case report," BMC Research Notes, vol. 11, no. 1, p. 165, 2018.

[24] D. Cattaneo, N. Perico, F. Gaspari, E. Gotti, and G. Remuzzi, "Glucocorticoids interfere with mycophenolate mofetil bioavailability in kidney transplantation," Kidney International, vol. 62, no. 3, pp. 1060-1067, 2002.

[25] N. Pourafshar, A. Karimi, X. Wen et al., "The utility of trough mycophenolic acid levels for the management of lupus 
nephritis," Nephrology, Dialysis, Transplantation, vol. 34, no. 1, pp. 83-89, 2019.

[26] A. Zabotti, M. Baraldo, L. Quartuccio, S. Sacco, G. De Marchi, and S. De Vita, "Optimizing the dose of mycophenolate mofetil for the maintenance treatment of lupus nephritis by therapeutic drug monitoring," Clinical Rheumatology, vol. 34, no. 1, pp. 171-174, 2015.

[27] S. Alexander, D. H. Fleming, B. S. Mathew et al., "Pharmacokinetics of concentration-controlled mycophenolate mofetil in proliferative lupus nephritis: an observational cohort study," Therapeutic Drug Monitoring, vol. 36, no. 4, pp. 423-432, 2014.

[28] P. Lertdumrongluk, P. Somparn, W. Kittanamongkolchai, O. Traitanon, S. Vadcharavivad, and Y. Avihingsanon, "Pharmacokinetics of mycophenolic acid in severe lupus nephritis," Kidney International, vol. 78, no. 4, pp. 389-395, 2010.

[29] T. Pawinski, "Therapeutic drug monitoring of mycophenolic acid: a potential treatment for lupus nephritis," Kidney International, vol. 78, no. 4, pp. 335-336, 2010.

[30] T. van Gelder, J. H. Berden, and S. P. Berger, "To TDM or not to TDM in lupus nephritis patients treated with MMF?," Nephrology, Dialysis, Transplantation, vol. 30, no. 4, pp. 560$564,2015$.

[31] W. Kittanamongkolchai, C. Rukrung, T. Supasiri et al., "Therapeutic drug monitoring of mycophenolate mofetil for the treatment of severely active lupus nephritis," Lupus, vol. 22, no. 7, pp. 727-732, 2013.

[32] T. Kiyokawa, H. Hanaoka, H. Iida et al., "High plasma mycophenolate acid concentration in the early phase of induction therapy predicts good renal outcome in lupus nephritis," Modern Rheumatology, vol. 30, no. 3, pp. 517-524, 2020.

[33] P. Luszczynska, T. Pawinski, P. K. Kunicki, M. Durlik, H. Augustyniak-Bartosik, and M. Hurkacz, "Pharmacokinetics of free and total mycophenolic acid in adult lupus nephritis patients-implications for therapeutic drug monitoring," European Journal of Clinical Pharmacology, vol. 75, no. 3, pp. 371379, 2019.

[34] I. Neumann, H. Fuhrmann, I. F. Fang, A. Jaeger, P. Bayer, and J. Kovarik, "Association between mycophenolic acid 12-h trough levels and clinical endpoints in patients with autoimmune disease on mycophenolate mofetil," Nephrology, Dialysis, Transplantation, vol. 23, no. 11, pp. 3514-3520, 2008.

[35] G. M. N. Daleboudt, M. E. J. Reinders, J. . Hartigh et al., “Concentration-controlled treatment of lupus nephritis with mycophenolate mofetil," Lupus, vol. 22, no. 2, pp. 171-179, 2013.

[36] N. Zahr, L. Arnaud, P. Marquet et al., "Mycophenolic acid area under the curve correlates with disease activity in lupus patients treated with mycophenolate mofetil," Arthritis and Rheumatism, vol. 62, no. 7, pp. 2047-2054, 2010. 